\title{
Propriedades de pasta de amidos de arroz nativo e acetilados
}

\author{
Pasting properties of native and acetylated rice starches
}

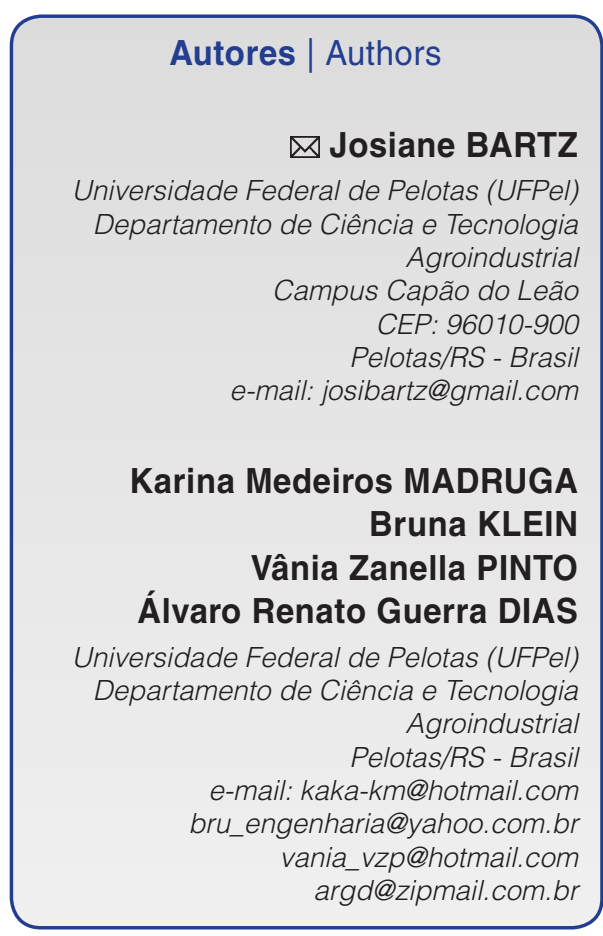

$\triangle$ Autor Correspondente / Corresponding Author Publicado / Published: dezembro/2012

\section{Resumo}

O amido de arroz apresenta características favoráveis a muitas aplicações industriais; no entanto, a natureza hidrofílica do amido na forma nativa pode apresentar algumas limitações para determinados tipos de processamento. Neste estudo, amido de arroz com médio teor de amilose foi acetilado sob catálise alcalina em duas condições reacionais para produzir acetatos de amido com diferentes graus de substituição (GS). A introdução de grupos acetila ao amido foi confirmada por espectroscopia de infravermelho com transformada de Fourier (FT-IV) e os acetatos de amido produzidos foram avaliados quanto às suas propriedades de pasta em viscoamilógrafo (RVA). A acetilação ocasionou reduções em todas as propriedades de pasta avaliadas (temperatura de pasta, viscosidade mínima, pico de viscosidade, viscosidade final e tendência à retrogradação), sendo a redução mais intensa no amido acetilado com maior GS.

Palavras-chave: Acetilação; Modificação química; Viscosidade; Retrogradação; RVA.

\section{Summary}

Rice starch has characteristics suitable to many industrial applications, however, the hydrophilic nature of the starch in native form may present some limitations for some uses. In this study, rice starch with medium amylose content was acetylated under alkaline catalysis on two reaction conditions to produce starch acetates with different degrees of substitution (DS). The introduction of acetyl groups to the starch was confirmed by infrared Fourier transform (FT-IR) and starch acetates produced were evaluated for their paste properties in viscoelastograf (RVA). Acetylation caused reductions in all properties paste (paste temperature, minimum viscosity, peak viscosity, final viscosity and retrogradation tendency) being the most intense reduction in acetylated starch with greater GS.

Key words: Acetylation; Chemical modification; Viscosity; Retrogradation; RVA. 


\section{Introdução}

O amido é uma fonte importante de carboidratos na dieta humana e amplamente utilizado na indústria de alimentos como espessante, estabilizante e agente de gelificação (GONZÁLEZ e PEREZ, 2002). A gelatinização do amido é tida como o colapso e a desintegração da ordem molecular dos grânulos, e conduz a modificações irreversíveis nas suas propriedades, tais como o inchamento do grânulo, a fusão da estrutura cristalina, a perda de birrefringência e a solubilização do amido (CEREDA, 2002; SINGH et al., 2007). De acordo com Cereda (2002), a gelatinização dos grânulos e a consequente formação de pasta dependem de vários fatores, como o tipo de amido, a concentração da dispersão, a temperatura utilizada e a intensidade de cisalhamento durante o aquecimento.

O amido de arroz na forma gelatinizada apresenta sabor suave e aspecto cremoso; além disso, o pequeno tamanho dos grânulos (2 a 10 m) fornece uma percepção de textura semelhante à da gordura (CHAMPAGNE, 1996). Adicionalmente, a ampla faixa no teor de amilose deste amido é um fator que aumenta as oportunidades para a sua utilização (TECHAWIPHARAT et al., 2008; ZHONG et al., 2009). De acordo com Techawipharat et al. (2008), o amido de arroz é classificado em função do seu teor de amilose, o qual pode variar entre 1 e $37 \%$, podendo ser considerado de baixo teor de amilose (12-20\%), de médio teor (20-25\%), de alto teor (>25\%) e com pouco ou isento de amilose (ceroso).

No entanto, a utilização de amidos na forma nativa pode apresentar algumas limitações no processamento de alimentos, como a inadequação das propriedades aos fins propostos, além da elevada tendência à separação de fases após a gelatinização, decorrente da reassociação das moléculas, com exsudação da água de hidratação durante o armazenamento (retrogradação e sinérese) (CEREDA, 2002; SINGH et al., 2007). Visando a superar essas deficiências, muitos estudos têm sido realizados com o intuito de buscar formas de modificar amidos de diferentes fontes botânicas (LAWAL, 2004; SODHI e SINGH, 2005; ADEBOWALE et al., 2005; BELLOPÉREZ et al., 2010).

Nesse aspecto, a acetilação pode diminuir a hidrofilicidade e melhorar as propriedades do amido (RAINA et al., 2007; SINGH et al., 2007). A acetilação constitui um método comum de modificação baseado na esterificação dos polímeros de amido com grupos acetila para formar acetatos de amidos (MARK e MEHLTRETTER, 1972). A substituição dos grupos hidroxilas por grupos acetila promove a redução nas interações entre as cadeias exteriores da amilopectina e entre as cadeias de amilose, conferindo novas características ao polímero (CHOl et al., 2009; BELLO-PÉREZ et al., 2010). Na medida em que a unidade de anidroglicose apresenta três hidroxilas disponíveis, as quais podem ser substituídas por grupos acetila, o grau de substituição (GS) máximo é de três unidades de grupos acetila por unidade de anidroglicose. Os acetatos de amido, de acordo com o GS, são classificados como de baixo GS $(<0,1)$, médio GS $(0,1-1,0)$ e de alto GS $(>1,0)$ (MARK e MEHLTRETTER, 1972).

A acetilação de amidos tem sido empregada na promoção da estabilidade e da resistência à retrogradação em amidos. Além disso, alterações como aumento na claridade de pasta, maior capacidade de absorção de água e redução da temperatura de gelatinização têm sido reportadas (SODHI e SINGH, 2005; CHOI et al., 2009; MBOUGUENG et al., 2012). Assim, considerando-se o potencial de uso comercial do amido de arroz, este estudo teve por objetivo avaliar a influência da acetilação com anidrido acético e da catálise alcalina nas propriedades de pasta de amido de arroz de médio teor de amilose.

\section{Material e métodos}

\subsection{Material}

Amido de arroz de médio teor de amilose (23,4\%), proveniente da cultivar Sasanishiki, foi extraído conforme o método proposto por Wang e Wang (2004). Os reagentes utilizados foram de grau analítico.

\subsection{Síntese dos acetatos de amido}

O amido de $\operatorname{arroz}(7,3 \%$ de umidade) foi acetilado a partir de dois métodos para produzir acetatos de amido com diferentes GS. Os métodos utilizados foram os propostos por Phillips et al. (1999), para obtenção de amido de baixo GS, e por Mark e Mehltretter (1972), para obtenção de amido com médio GS (1972).

No método proposto por Phillips et al. (1999), o amido de $\operatorname{arroz}(100 \mathrm{~g})$ foi disperso em $225 \mathrm{~mL}$ de água destilada, sendo a suspensão submetida à agitação de $1500 \mathrm{rpm}$ durante $60 \mathrm{~min} / 24^{\circ} \mathrm{C} . \mathrm{O} \mathrm{pH}$ da suspensão foi ajustado para 8,0 com solução aquosa de $\mathrm{NaOH} 3,0 \%$, sendo $6,0 \mathrm{~g}$ de anidrido acético adicionados lentamente à suspensão, mantendo-se o pH entre 8,0 e 8,4, com auxílio da solução alcalina. Após completa adição do anidrido acético, a reação prosseguiu por 10 minutos e foi então interrompida pelo ajuste de $\mathrm{pH}$ para 4,5 (solução de $\mathrm{HCl}$ 0,5 N). A suspensão final foi centrifugada durante 3 minutos a $1000 \mathrm{~g}$ e submetida a lavagens sucessivas com água e a uma lavagem final com álcool etílico 95\%.

O outro procedimento adotado (MARK e MEHLTRETTER, 1972) consisitiu em realizar a reação sob condição anidra, em que $23 \mathrm{~g}$ de amido foram dispersos em $74 \mathrm{~mL}$ de anidrido acético e acrescidos de 2,9 mL de solução de $\mathrm{NaOH} 50 \%$ como catalisador. A reação foi conduzida a $120^{\circ} \mathrm{C}$ em banho de glicerol e sob agitação 
de 1200 rpm. Decorrido o tempo, $100 \mathrm{~mL}$ de álcool etílico absoluto foram adicionados, sendo a amostra filtrada em funil de Büchner e lavada com porções de $50 \mathrm{~mL}$ de álcool etílico.

Os acetatos de amido produzidos foram secos a $40{ }^{\circ} \mathrm{C}$ por 24 horas em estufa com circulação de ar.

\subsection{Grau de substituição (GS) e percentual de grupos acetila (\% Ac)}

O percentual de grupos acetila (\% Ac) (Equação 1) e o grau de substituição (GS) (Equação 2) foram determinados por titulometria, conforme proposto por Würzburg (1986). Aproximadamente $1 \mathrm{~g}$ de cada amostra foi adicionado a frascos de vidro com tampa e acrescido de $50 \mathrm{~mL}$ de álcool etílico a $75 \%$. As amostras foram aquecidas em banho-maria a $50{ }^{\circ} \mathrm{C}$ por 30 minutos e, após resfriadas, foram adicionadas de $40 \mathrm{~mL}$ de $\mathrm{KOH}$ $0,5 \mathrm{~N}$ e mantidas sob agitação de $200 \mathrm{rpm}$ por 72 horas. Decorrido o tempo, o excesso de álcali foi titulado com $\mathrm{HCl} 0,5 \mathrm{~N}$, utilizando-se fenolftaleína como indicador. A solução neutralizada foi mantida sob agitação por 2 horas e o excesso de álcali, o qual pode ter lixiviado da amostra, foi titulado. Uma amostra em branco com o amido nativo também foi realizada.

$\% A c=\frac{[(\mathrm{ml} \text { branco }-\mathrm{ml} \text { amostra }) \times \mathrm{MHCl} \times 0,043 \times 100]}{\text { peso da amostra }(\mathrm{g})}$

O grau de substituição é definido como o número médio de sítios por unidade de anidroglicose que recebeu um grupo substituinte.

$$
\mathrm{GS}=\frac{(162 \times \% \mathrm{Ac})}{[4300-(42 \times \% \mathrm{Ac})]}
$$

\subsection{Espectroscopia no infravermelho com transformada de Fourier (FT-IV)}

Os espectros de infravermelho das amostras de amidos de arroz nativo e acetilados foram obtidos em espectrofotômetro com transformada de Fourier (IR Prestige-21; Shimadzu) na região de $4000-400 \mathrm{~cm}^{-1}$. Foram preparadas pastilhas a partir da mistura das amostras com $\mathrm{KBr}$ em uma proporção de 1:100 (amostra: $\mathrm{KBr}$ ). Foram recolhidas dez leituras a uma resolução de $4 \mathrm{~cm}^{-1}$.

\subsection{Propriedades de pasta}

As propriedades viscoamilográficas dos amidos foram avaliadas por RVA - Rapid Visco Analyser (modelo RVA-4, Newport Scientific, Austrália), por meio do perfil Standard Analysis 1 utilizando-se 2,5 g de amostra corrigida para $14 \%$ de umidade. As amostras foram aquecidas a $50{ }^{\circ} \mathrm{C} \mathrm{em} 1$ minuto e, posteriormente, a $95{ }^{\circ} \mathrm{C}$ em 3,5 minutos, sendo mantidas a $95{ }^{\circ} \mathrm{C}$ durante
2,5 minutos. A seguir, foram resfriadas para $50{ }^{\circ} \mathrm{C} \mathrm{em}$ 3,8 minutos e mantidas a $50^{\circ} \mathrm{C}$ por 2 minutos. A velocidade de rotação foi mantida a $960 \mathrm{rpm}$ durante 10 segundos e então mantida a 160 rpm durante o restante do processo. Foram avaliadas a temperatura de início de formação de pasta, a viscosidade máxima, a viscosidade mínima, a quebra da viscosidade, a viscosidade final e a tendência à retrogradação.

\subsection{Análise estatística}

As análises foram realizadas em triplicata e os resultados foram submetidos à análise de variância (ANOVA) e à comparação de médias pelo teste de Tukey, com nível de $5 \%$ de significância.

\section{Resultados e discussão}

Os acetatos de amido de arroz produzidos apresentaram GS de 0,01 $\pm 0,00$ e 0,67 $\pm 0,02$ (Tabela 1), o que os classificam como de baixo $(<0,1)$ e de médio $(0,1-1,0)$ GS, respectivamente. Amidos acetilados com baixo GS são comumente empregados na indústria alimentícia como promotores de consistência, textura e estabilidade nos alimentos, enquanto que amidos de GS intermediários ou altamente substituídos encontram aplicação como termoplásticos substitutos dos acetatos de celulose (BISWAS et al., 2008). Além disso, mais recentemente, acetatos de amido (com baixo e alto GS) têm sido sugeridos como materiais biodegradáveis na elaboração de embalagens alimentícias e em diversas aplicações farmacêuticas (BISWAS et al., 2008; CHI et al., 2008).

Na Figura 1, são apresentadas as mudanças ocorridas na estrutura química das moléculas do amido de arroz após a acetilação por espectroscopia de infravermelho. A confirmação da acetilação, bem como a extensão da incorporação dos grupos acetila à molécula do amido, podem ser observadas pelo aparecimento de um pico na região de absorção de $1743 \mathrm{~cm}^{-1}$, referente ao grupo da carbonila $(\mathrm{C}=\mathrm{O})$ dos grupos acetila esterificados. Pode-se observar que, conforme aumenta o GS dos acetatos, a intensidade do pico na região de $1743 \mathrm{~cm}^{-1}$ também aumenta, assim como ocorre redução da intensidade do pico na região de absorção dos grupos hidrofílicos $(\mathrm{OH})$ em $3450 \mathrm{~cm}^{-1}$ e $1641 \mathrm{~cm}^{-1}$. O espectro

Tabela 1. Percentual de grupos acetila (\% Ac) e grau de substituição (GS) dos acetatos de amido de arroz.

\begin{tabular}{lccr} 
& \multirow{2}{*}{ Nativo } & \multicolumn{2}{c}{ Acetilados } \\
& & Baixo GS $^{\mathbf{A}}$ & ${\text { Médio } \mathbf{G S}^{\mathbf{B}}}$ \\
\hline \% AC & - & $0,21 \pm 0,01^{\mathrm{a}^{*}}$ & $14,13 \pm 0,42^{\mathrm{b}}$ \\
GS & - & $0,01 \pm 0,00^{\mathrm{a}}$ & $0,67 \pm 0,02^{\mathrm{b}}$ \\
\hline
\end{tabular}

*Médias ( \pm desvio padrão) seguidas por letras diferentes na mesma linha diferem estatisticamente $(p<0,05)$. ${ }^{A}$ Baixo $G S=$ Método de Phillips et al. (1999); ${ }^{B}$ Método de Mark e Mehltretter (1972). 
do amido de arroz nativo mostra picos intensos em números de onda em torno de $1018 \mathrm{~cm}^{-1}, 1080 \mathrm{~cm}^{-1} \mathrm{e}$ $1157 \mathrm{~cm}^{-1}$, atribuídos à vibração da ligação C-O. Também foram registrados picos em $1641 \mathrm{~cm}^{-1}$, relativos à água fortemente ligada, em $3450 \mathrm{~cm}^{-1}$, resultante da vibração dos grupos hidrofílicos hidroxilas $(\mathrm{O}-\mathrm{H})$, e em $2960 \mathrm{~cm}^{-1}$, derivados da vibração do estiramento $\mathrm{C}-\mathrm{H}$ da molécula de anidroglicose.

As propriedades viscoamilográficas dos amidos de arroz nativo e acetilados podem ser visualizadas na Tabela 2. Os acetados de amido produzidos apresentaram significativas alterações nas suas propriedades de pasta em relação à sua contraparte nativa e entre si, sendo o nível de alteração mais intenso no acetato de amido de GS mais elevado.

A temperatura de pasta para o amido de arroz nativo apresentou valor médio de $62,95 \pm 0,05{ }^{\circ} \mathrm{C}$ e não diferiu estatisticamente $(p<0,05)$ do amido acetilado de baixo
GS $\left(62,02 \pm 1,24^{\circ} \mathrm{C}\right)$, mas foi significativamente superior à temperatura necessária para a formação de pasta no acetato de médio GS $\left(60,35 \pm 0,01^{\circ} \mathrm{C}\right)$. Uma redução na temperatura de pasta após a acetilação é esperada, visto que a presença dos grupos acetila contribui para a desorganização da estrutura intragranular, resultando na redução das forças associativas na região amorfa dos grânulos e interferindo nas ligações de hidrogênio intra e intermoleculares que estabilizam a estrutura (RAINA et al., 2007). Observações similares são reportadas por Adebowale et al. (2005) em amido de jaca com 0,23 GS, e por Sodhi e Singh (2005) em amidos de arroz de diferentes cultivares (0,09-0,144 GS).

A Figura 2 representa o perfil viscoamilográfico obtido durante o aquecimento e o resfriamento de suspensões aquosas dos amidos nativo e acetilados. 0 pico de viscosidade, a viscosidade mínima, a quebra da viscosidade e a viscosidade final foram as propriedades de



Figura 1. Espectro de FT-IV dos amidos nativo e acetilados.

Tabela 2. Propriedades viscoamilográficas dos amidos de arroz nativo e acetilados.

\begin{tabular}{|c|c|c|c|}
\hline & \multirow{2}{*}{ Nativo } & \multicolumn{2}{|c|}{ Acetilados } \\
\hline & & Baixo GS*** & Médio GS \\
\hline Temperatura de pasta $\left(\mathrm{T}^{\circ} \mathrm{C}\right)$ & $62,95 \pm 0,05^{a \star}$ & $62,02 \pm 1,24^{a b}$ & $60,35 \pm 0,01^{b}$ \\
\hline Pico de viscosidade (RVU) & $283,47 \pm 1,37^{a}$ & $232,25 \pm 2,12^{b}$ & $68,08 \pm 0,23^{c}$ \\
\hline Viscosidade mínima (RVU) & $219,08 \pm 6,25^{\mathrm{a}}$ & $174,83 \pm 5,42^{b}$ & $63,33 \pm 0,01^{c}$ \\
\hline Quebra (RVU) & $64,39 \pm 5,34^{a}$ & $57,41 \pm 3,30^{a}$ & $4,75 \pm 0,24^{b}$ \\
\hline Viscosidade final (RVU) & $360,03 \pm 5,34^{a}$ & $318,54 \pm 0,27^{b}$ & $183,00 \pm 0,24^{c}$ \\
\hline Retrogradação (RVU**) & $142,45 \pm 0,53^{a}$ & $140,08 \pm 0,01^{b}$ & $119,66 \pm 0,23^{c}$ \\
\hline
\end{tabular}

*Médias aritméticas ( \pm desvio padrão) seguidas por letras diferentes na mesma linha diferem estatisticamente $(p<0,05)$. ${ }^{*}$ RVU: Rapid Visco Unit. ${ }^{* *}$ Baixo GS = 0,01; Médio GS = 0,67. 




Figura 2. Perfil viscoamilográfico (RVA) dos amidos de arroz nativo e acetilados.

pasta que apresentaram maior redução após a acetilação. Os acetatos de amido diferiram significativamente do amido nativo e entre si, sendo tanto maior a redução das propriedades viscoamilográficas quanto maior o GS obtido. O pico de viscosidade do amido nativo diminuiu de $283,47 \pm 1,37$ para $232,25 \pm 2,12$ e para $68,08 \pm 0,23$ nos acetatos de amido com 0,01 e 0,67 GS, respectivamente.

Comumente, após a modificação química por acetilação, o pico de viscosidade apresenta uma tendência a subir com o aumento do GS, o que, segundo Singh et al. (2007), está relacionado com o possível aumento na capacidade de absorção e retenção de água pelos substituintes introduzidos. Em estudos com amido de arroz realizados por Sodhi e Singh (2005) e por Raina et al. (2007), os autores reportaram um aumento na viscosidade de pasta de amido de arroz comercial após a acetilação, independentemente do conteúdo de amilose e da cultivar avaliada. No entanto, a redução na viscosidade de pasta, observada neste estudo, pode ser devida à despolimerização das cadeias de amido em razão da ocorrência de hidrólises secundárias durante o processo reacional. Resultados semelhantes são reportados por Lawal (2004) em amido de taioba com GS de 0,30, por Bello-Pérez et al. (2010) em amido de cevada com GS de 0,9 e 2,7, e por Choi et al. (2009) em amido de milho com GS entre 0,046 e 0,089.

A viscosidade mínima dos amidos acetilados, em relação ao nativo, apresentou percentuais de redução de $20 \%$ no acetato com 0,01 GS e de $71 \%$ no acetato de médio GS $(0,67)$. Diversamente, a redução na viscosidade final foi de $11,5 \%$ e $49 \%$ nos acetatos de baixo e médio GS, respectivamente. Redução expressiva nas viscosidades mínima e final, como observada neste estudo, é também mencionada por Adebowale et al. (2005) em amido de jaca (61\% para viscosidade mínima e $15 \%$ para viscosidade final). No entanto, Sodhi e Singh (2005), ao avaliarem amidos de arroz acetilados de diferentes cultivares, observaram efeito contrário e reportam um aumento em ambas as viscosidades após a modificação. Por sua vez, Raina et al. (2007), também em estudo de acetilação com amido de diferentes cultivares de arroz, citam um aumento na viscosidade mínima, tal como Sodhi e Singh (2005), mas redução na viscosidade final, assim como o observado neste estudo.

O valor de quebra para o amido nativo de arroz foi $64,39 \pm 5,34$, diminuindo para 57,41 $\pm 3,30$ no acetato de baixo GS, e para 4,75 $\pm 0,24$ no acetato de médio GS. Assim como a quebra, a tendência à retrogradação foi consideravelmente atenuada após a modificação, sendo mais expressiva no acetato de amido de maior GS final. Essa redução na resistência da pasta às ações térmica e mecânica, bem como na tendência à retrogradação, vai ao encontro de outros estudos com acetilação de amido sob condições semelhantes (RAINA et al., 2007; SINGH et al., 2007; BELLO-PÉREZ et al., 2010). Segundo Singh et al. (2007), a introdução dos substituintes acetila às cadeias do amido restringe a reassociação das moléculas após o resfriamento da pasta, proporcionando menores valores de retrogradação. 


\section{Conclusão}

A acetilação interfere significativamente nas propriedades viscomilográficas de amido de arroz de médio teor de amilose. Após a modificação, ocorre redução na temperatura de pasta, na viscosidade mínima, no pico de viscosidade, na viscosidade final e na tendência à retrogradação, sendo o nível de redução mais intenso no acetato de amido de maior grau de substituição (GS). Amidos com características de interesse para muitas aplicações industriais, tais como pastas mais resistentes à retrogradação, menos viscosas e com menor resistência a ação mecânica, podem ser obtidos por acetilação, sendo a intensidade dessas alterações proporcional à quantidade de grupos acetila inserida no amido.

\section{Agradecimentos}

À CAPES, pelo suporte financeiro.

\section{Referências}

ADEBOWALE, K. O.; OLU-OWOLABI, B. I.; OLAWUMI, E. K.; LAWAL, O. S. Functional properties of native, physically and chemically modified breadfruit (Artocarpus artilis) starch. Industrial Crops and Products, Amsterdan, v. 21, n. 3, p. 343-351, 2005. http://dx.doi.org/10.1016/j. indcrop.2004.05.002

BELLO-PÉREZ, L. A.; AGAMA-ACEVEDO, E.; ZAMUDIO-FLORES, P. B.; MENDEZ-MONTEALVO, G.; RODRIGUEZ-AMBRIZ, S. L. Effect of low and high acetylation degree in the morphological, physicochemical and structural characteristics of barley starch. LWT - Food Science and Technology, London, v. 43, n. 9, p. 1434-1440, 2010. http://dx.doi.org/10.1016/j.Iwt.2010.04.003

BISWAS, A.; SHOGREN, R. L.; SELLING, G.; SALCH, J.; WILLETT, J. L.; BUCHANAN, C. M. Rapid and environmentally friendly preparation of starch esters. Carbohydrate Polymers, Barking, v. 74, n. 1, p. 137-141, 2008. http://dx.doi.org/10.1016/j. carbpol.2008.01.013

CEREDA, M. P. Propriedades Gerais do Amido. São Paulo: Fundação Cargill, 2002. 221 p. (Série: Culturas de Tuberosas Amiláceas Latino-americanas, v. 1).

CHAMPAGNE, E. T. Rice starch composition and characteristics. Cereal Foods World, Minneapolis, v. 41, p. 833-838, 1996.

CHI, H.; XU, K.; CHEN, Q.; XUE, D.; SONG, C.; ZHANG, W.; WANG, P. Effect of acetylation on the properties of corn starch. Food Chemistry, London, v. 106, n. 3, p. 923-928, 2008. http:// dx.doi.org/10.1016/j.foodchem.2007.07.002

CHOI, H-S.; KIM, H-S.; PARK, C-S.; KIM, B-Y.; MAIK, M-Y. Ultra high pressure (UHP)-assisted acetylation of corn starch. Carbohydrate Polymers, Barking, v. 78, n. 4, p. 862-868, 2009. http://dx.doi.org/10.1016/j.carbpol.2009.07.005
GONZÁLEZ, Z.; PÉREZ, E. Effect of acetylation on some properties os rice starch. Starch - Stärke, Weinheim, v. 54, n. 3-4, p. 148-154, 2002. http://dx.doi.org/10.1002/1521379X(200204)54:3/4<148::AID-STAR148>3.0.CO;2-N

LAWAL, O. S. Composition, physicochemical properties and retrogradation of native, oxidized, acetylated and acidthinned new cocoyam (Xanthosoma sagittifolium) starch. Food Chemistry, London, v. 87, n. 2, p. 205-218, 2004. http://dx.doi. org/10.1016/j.foodchem.2003.11.013

MARK, A. M.; MEHLTRETTER, C. L. Facile preparation of starch triacetates. Starch - Stärke, Weinheim, v. 24, n. 3, p. 73-76, 1972. http://dx.doi.org/10.1002/star.19720240302 http://dx.doi.org/10.1002/star.19720240302

MBOUGuenG, P. D.; TENIN, D.; SCHER, J.; TCHIÉGANG, C. Influence of acetylation on physicochemical, functional and thermal properties of potato and cassava starches. Journal of Food Engineering, Essex, v. 108, n. 2, p. 320-326, 2012. http:// dx.doi.org/10.1016/j.jfoodeng.2011.08.006

PHILLIPS, D. L.; LIU, H.; PAN, D.; CORKE, H. General application of Raman spectroscopy for the determination of level of acetylation in modified starches. Cereal Chemistry, Saint Paul, v. 76, n. 3, p. 439-443, 1999. http://dx.doi.org/10.1094/ CCHEM.1999.76.3.439

RAINA, C. S.; SINGH, S.; BAWA, A. S.; SAXENA, D. C. A comparative study of Indian rice starches using different modification model solutions. LWT - Food Science and Technology, London, v. 40, n. 5, p. 885-892, 2007. http://dx. doi. org/10.1016/j.Iwt.2006.03.025

SODHI, N. S.; SINGH, N. Characteristics of acetylated starches prepared using starches separated from different rice cultivars. Journal of Food Engineering, Essex, v. 70, n. 1, p. 117-127, 2005. http://dx.doi.org/10.1016/j.jfoodeng.2004.09.018

SINGH, J.; KAUR, L.; McCARTHY, O. J. Factors influencing the physic-chemical, morphological, thermal and rheological properties of some chemically modified starches for food apllications - A review. Food Hydrocolloids, Oxford, n. 1, v. 21, p. 1-22, 2007. http://dx.doi.org/10.1016/j.foodhyd.2006.02.006

TECHAWIPHARAT, J.; SUPHANTHARIKA, M.; BEMILLER, J. N. Effects of cellulose derivatives and carrageenans on the pasting, paste, and gel properties of rice starches. Carbohydrate Polymers, Barking, v. 73, n. 3, p. 417-426, 2008.

WANG, L.; WANG, Y. J. Rice starch isolation by neutral protease and high-intensity ultrasound. Journal of Cereal Science, London, v. 39, n. 2, p. 291-296, 2004. http://dx.doi.org/10.1016/j. jcs.2003.11.002

WÜRZBURG, O. B. Modified Starches: Properties and Uses. Boca Ratón: CRC Press, 1986. 277 p. PMid:3703392.

ZHONG, F.; LI, Y.; IBANZ, A. M.; OH, M. H.; McKENZIE, K. S.; SHOEMAKER, C. The effect of rice variety and starch isolation method on the pasting and rheological properties of rice starch pastes. Food Hydrocolloids, Oxford, v. 23, n. 2, p. 406-414, 2009. http://dx.doi.org/10.1016/j.foodhyd.2008.02.003 